CCE - COORDENAÇÃO CENTRAL DE EXTENSÃO

Pós Graduação em Avaliação Neuropsicológica

\author{
Rosane do Rêgo Lopes Fonseca
}

\title{
A IMPORTÂNCIA DA AVALIAÇÃO NEUROPSICOLÓGICA PARA O DIAGNÓSTICO PRECOCE DO COMPROMETIMENTO COGNITIVO LEVE
}

Orientadora: Helenice Charchart Fichman 


\title{
CCE - COORDENAÇÃO CENTRAL DE EXTENSÃO \\ Pós Graduação em Avaliação Neuropsicológica
}

\author{
Rosane do Rêgo Lopes Fonseca
}

\section{A IMPORTÂNCIA DA AVALIAÇÃO NEUROPSICOLÓGICA PARA O DIAGNÓSTICO PRECOCE DO COMPROMETIMENTO COGNITIVO LEVE}

\begin{abstract}
Monografia apresentada ao Programa de Pós-Graduação em Avaliação Neuropsicológica da PUC-Rio como requisito parcial para obtenção do título de Especialista em Avaliação Neuropsicológica. Aprovada pela Comissão Examinadora abaixo assinada.
\end{abstract}

Orientadora: Helenice Charchart Fichman

Rio de Janeiro Setembro 2018 


\section{SUMÁRIO}

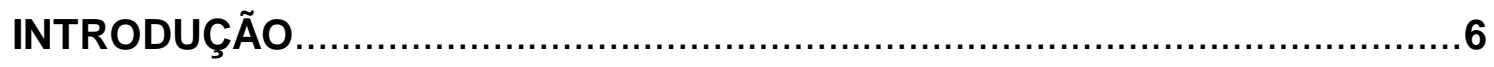

CAPÍTULO I - A IMPORTÂNCIA DA AVALIAÇÃO NEUROPSICOLÓGICA ...8

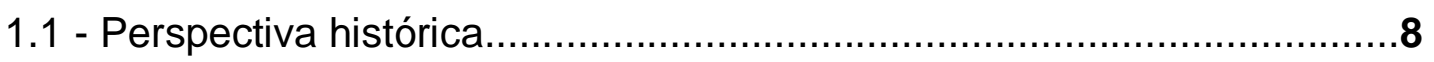

1.2 - A Avaliação neuropsicológica........................................................11

CAPÍTULO II - O COMPROMETIMENTO COGNITIVO LEVE......................19

2.1 - Alguns critérios importantes para fazer o diagnóstico...........................23

2.2 - O Diagnóstico de Comprometimento Cognitivo Leve............................25

CAPÍTULO III -O COMPROMETIMENTO COGNITIVO LEVE E AVALIAÇÃO

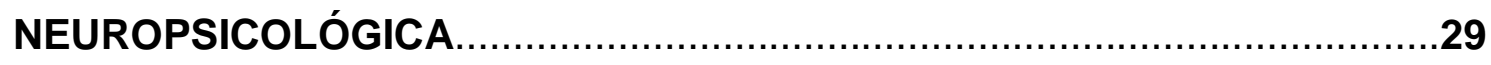

3.1 - Os subtipos Amnéstico e não Amnéstico............................................29

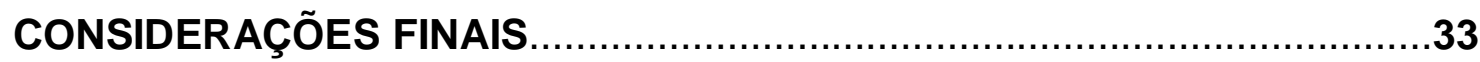

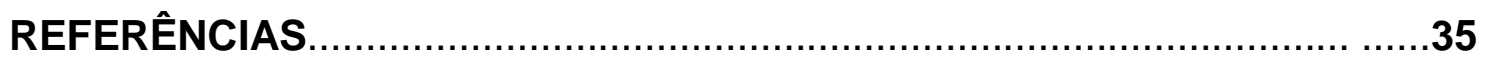

TABELAS:

Tabela 1 - Sistemas de Memória por Budson. 


\section{RESUMO}

Esta monografia trata de ressaltar e analisar o tema Comprometimento Cognitivo Leve (CCL) e o seu diagnóstico precoce no sentido também de destacar a importância da avaliação neuropsicológica para o idoso, pois percebe-se que a cognição aparece na queixa subjetiva como na evidência objetiva, assim os testes utilizados no diagnóstico do CCL, pode apontar para uma perda de memória, mas não necessariamente o Alzheimer. Entende-se assim a relevância da avaliação neuropsicológica para os pacientes idosos. Portanto, para a execução desta monografia, foi utilizado referenciais teóricos que contribuíram para esse estudo e especificamente, foi realizada uma revisão bibliográfica de literatura por meio de um recorte de textos que abordam a questão.

Palavras chave: CCL, avaliação neuropsicológica, diagnóstico, Alzheimer. 


\section{ABSTRACT}

This monograph tries to emphasize and analyze the subject Light Cognitive Impairment (CCL) and its early diagnosis in the sense of also highlighting the importance of the neuropsychological evaluation for the elderly, because it is perceived that the cognition appears in the subjective complaint as in the objective evidence, so the tests used in the diagnosis of CCL may point to a memory loss, but not necessarily Alzheimer's. The relevance of neuropsychological assessment for elderly patients is thus understood. Therefore, for the execution of this monograph, we used theoretical references that contributed to this study and specifically, a bibliographical review of the literature was carried out by means of a clipping of texts that approach the question.

Keywords: CCL, neuropsychological evaluation, diagnosis, Alzheimer. 


\section{Introdução}

A presente monografia de conclusão de curso traz o tema "A importância da Avaliação Neuropsicológica para o diagnóstico precoce do comprometimento Cognitivo Leve", que objetiva destacar o papel da avaliação neuropsicológica, que investigará diferenças nas habilidades cognitivas e que contribuem para uma identificação das diversas áreas funcionais de transição entre o envelhecimento benigno e as síndromes demenciais. A presente proposta de estudo justifica-se pelo fato de conhecer a aplicabilidade do diagnóstico diferencial de Comprometimento Cognitivo Leve e desenvolver um estudo acerca do assunto, através de uma pesquisa minuciosa de recortes de livros e artigos, na busca de aprofundar algumas ideias que ainda são vagas. E especificamente nessa atividade de pesquisa, tais ideias irem clareando aos poucos, não totalmente fechadas, mas no exercício e na prática de realizar um estudo sobre a Doença de Alzheimer que de acordo com o Manual Diagnóstico e Estatístico de Transtornos Mentais (DSM-IVTR), essa doença é caracterizada pela perda de memória associada com outras alterações cognitivas e prejuízos no desempenho das atividades de vida diária (American Psychiatric Association - APA, 2004).

No final de todo o trabalho, teremos um resultado do esforço de pesquisa de campo, de leitura, de experimentação e reflexão.

E é a partir dessa investigação sobre o diagnóstico que é um diferencial para o Comprometimento Cognitivo Leve (CCL), que iremos entender que a Doença de Alzheimer possui um declínio contínuo e progressivo dos estados normais de consciência, comprometendo o indivíduo em seu funcionamento, assim podemos compreender a importância da avaliação neuropsicológica.

Como metodologia, para o desenvolvimento deste trabalho cabe ressaltar a concordância com os pressupostos de alguns autores sobre o estudo do Comprometimento Cognitivo Leve e seu diagnóstico. Partindo do princípio que "não há metodologias 'boas' ou 'más' em si, e sim metodologias adequadas ou inadequadas para tratar um determinado problema". 
Diante de um estudo de cunho qualitativo e quantitativo e com viés exploratório, para alcançar os objetivos propostos serão percorridas as seguintes etapas:

O trabalho consiste no levantamento de material bibliográfico, que possibilitará uma visão panorâmica sobre o tema e sobre a área em estudo. Portanto, esse estudo vem para articular e conversar com essas questões.

Desta forma apresentaremos o capítulo I com a importância da avaliação neuropsicológica para os estados neurológicos e fins de diagnóstico.

O capítulo II trará os conceitos de Comprometimento Cognitivo Leve (CCL) e sua definição.

O capítulo III, será apresentado uma articulação que trabalhamos nos capítulos anteriores.

Seguindo com as considerações finais e as referências bibliográficas. 


\section{Capítulo I - A importância da avaliação neuropsicológica}

Esse capítulo irá nos mostrar a importância de uma avaliação neuropsicológica, mas para isso é preciso antes de tudo conhecer melhor o que vem a ser a neuropsicologia e seu contexto histórico. Então para que isso aconteça de partida vamos à definição do termo que surgiu no século $\mathrm{XX}$, e seguidamente passamos para a avaliação neuropsicológica.

A preocupação com as inter-relações entre cérebro e mente vem desde os egípcios. Hoje temos tomografia computadorizada e Ressonância Magnética, técnicas que combinadas à avaliação neuropsicológica representam um enorme avanço para o diagnóstico e tratamento de inúmeras patologias.

Neuropsicologia é a ciência dedicada a estudar a expressão comportamental das disfunções cerebrais, segundo a definição de Muriel D. Lezak: Avaliação Neuropsicológica é o método para investigação do funcionamento cerebral através do estudo comportamental. Os objetivos da avaliação neuropsicológica são basicamente auxiliar o diagnóstico diferencial, estabelecer a presença ou não de disfunção cognitiva e o nível de funcionamento em relação ao nível ocupacional, localizar alterações sutis, a fim de detectar as disfunções ainda em estágios iniciais. Contribui para planejamento do tratamento e no acompanhamento da evolução do quadro em relação aos tratamentos medicamentoso, cirúrgico e reabilitação. Difere da avaliação psicológica por tomar como ponto de partida o cérebro.

Portanto, dos recursos utilizados nas avaliações neuropsicológicas são diversos, baseados principalmente em material desenvolvido em laboratórios de neuropsicologia e neurologia. Estas duas ciências, embora distintas na história, hoje contribuem fundamentando a prática na avaliação neuropsicológica.

\section{1 - Perspectiva histórica}

É com esta perspectiva histórica que compreendemos então o avanço na avaliação das funções cognitivas relacionadas ao cérebro. O termo Neuropsicologia propriamente 
dito apareceu apenas no século XX, pelas palavras do Sir William Osler (1913), este utilizou o termo Neuropsychology numa exposição no Johns Hopkins Hospital.

Lashley (1938) desenvolveu a teoria equipotencial para a qual as alterações do comportamento dependiam mais de quantidade de massa que localização. Esta posição iria influenciar as teorias da Gestalt, no entanto, a prática hoje mostra o contrário. MacLean (1952) introduziu o termo sistema límbico e contribuiu para o estudo das especializações hemisféricas. Teuber (1955), Weiskrantz (1968) e Shallice (1979), através de suas pesquisas, mostraram a independência de tipos específicos de processamento da informação, evidenciando a dissociação das funções.

Na segunda metade do século XX, o neuropsicólogo americano Ward Halstead (1947) pesquisou testes mais sensíveis para aquilo que ele acreditava ser o substrato biológico da inteligência. Selecionou dez testes sensório-motores para a sua bateria neuropsicológica. Seu seguidor Ralph Reitan (1955) selecionou sete dos dez testes (funções sensoriais, de percepção, motoras, verbais e abstração) e completou a bateria com WAIS e Minnesota Multiphasic Personality Inventory (MMPI). Alguns destes testes são ainda muito utilizados, como o Category Test e o Trail Making Test.

"Inteligência é o Inter jogo dinâmico entre as três unidades com a ativação, regulação e planejamento do ato consciente pelos lobos frontais". Com esta proposição o russo Alexander Luria (1966) elaborou a teoria dos Sistemas Funcionais: 1) Unidade de Atenção (sistema reticular) 2) Unidade Sensorial (áreas primárias, secundárias e terciárias) 3) Unidade de Planejamento (áreas primárias, secundárias e terciárias). Desenvolveu vários métodos de eliciar comportamentos e assim analisá-los qualitativamente. A contribuição de Luria veio a incrementar uma visão mais dinâmica do funcionamento cerebral, e colaborar na compreensão do funcionamento das áreas frontais. Por algum tempo o trabalho desenvolvido na Rússia ficou distante do mundo ocidental, mas posteriormente alcançou projeção mundial. No entanto, pouco se sabe sobre o trabalho desenvolvido com testes quantitativos no Instituto Bekhterev de Psiquiatria e Psicologia Médica de St. Petesburg.

Dra. Elisabeth Warrington (Inglaterra) enfocou basicamente a análise das funções cognitivas relacionadas às disfunções cerebrais. Propôs a síntese dos métodos intuitivo e 
empírico resultando na análise de habilidades complexas, seus componentes e subcomponentes (1990). Sua bateria de testes neuropsicológicos contém testes de inteligência geral, percepção visual, funções verbais, memória para eventos e raciocínio. Elaborou vários testes de memória visual, verbal, pesquisando a diferenciação entre evocação e reconhecimento, percepção visual, e também estudos detalhados sobre as correlações neuropatológicas dos subtestes do WAIS R.

Arthur Benton (EUA) fez importantes contribuições para os métodos de avaliação neuropsicológica, desenvolveu testes de memória, de percepção visual e práxis construtivas. Edith Kaplan e o grupo de Boston (EUA) desenvolveram métodos de avaliação do processamento das informações nos diversos testes quantitativos. Elaboraram em 1991 o WAIS R NI, onde compilaram uma série de experiências sobre a avaliação das respostas do WAIS R fora dos limites de tempo do manual. Esta forma "Neuropsychological Instrument" (NI) traz significativa contribuição para a compreensão do funcionamento dos pacientes que não conseguem responder dentro das regras do teste. A análise das construções dos Cubos (Block Design) contribui para a diferenciação entre lesões. Kaplan utiliza uma bateria básica com testes satélites de inteligência, conceituação, memória, linguagem, percepção visual, habilidades acadêmicas e funções motoras.

Também não podemos deixar de mencionar a contribuição de Brenda Milner (1975), do Montreal Neurological Institute, no estudo dos pacientes epilépticos submetidos à cirurgia dos lobos temporal e frontal. Através da análise dos resultados dos testes de Wada e dos estudos neuropsicológicos pré e pós-cirúrgicos, Milner desenvolveu testes para avaliação de memória e funções executivas.

Década 90, estamos na Década do Cérebro. A Neuropsicologia, embora tenha suas raízes na antiguidade vem evoluindo no mundo inteiro. Os exames por imagem evoluíram significativamente, temos Tomografia Computadorizada (desde 1973) e Ressonância Magnética (desde 1985) acessíveis para diagnóstico mais preciso de lesões cerebrais. Os diversos centros de pesquisa, hoje mais especializados, desenvolvem trabalhos sobre envelhecimento normal e patológico, distúrbios cognitivos nas epilepsias, alterações de raciocínio nos pacientes psiquiátricos, distúrbios de 
aprendizagem, distúrbios da fala e outras funções cognitivas relacionadas às diferentes patologias do Sistema Nervoso Central. A Neuropsicologia voltou seus interesses para as correlações com os exames de imagem, estudos aprofundados das funções corticais e determinação mais precisa do nível de função.

Nos dias atuais devido à divulgação e o interesse crescente neste campo, não é possível abarcar aqui todos os neuropsicólogos e neurologistas que contribuíram e ainda contribuem significativamente. Mencionamos aqui o trabalho de peso de Marsel Mesulam e Norman Geschwind na neurologia comportamental.

Em 1989 foi fundada em Buenos Aires a Sociedade Latino Americana de Neuropsicologia, em 1991, por ocasião do II Congresso Latino Americano de Neuropsicologia e do I Congresso Brasileiro de Neuropsicologia, foi organizada a Sociedade Brasileira de Neuropsicologia, órgão atuante na divulgação desta ciência no nosso país e proporcionaram condições para formação de profissionais.

\section{2 - A Avaliação Neuropsicológica}

Conforme os pressupostos e matrizes básicas mencionadas, entendemos que a avaliação neuropsicológica utiliza testes psicométricos e neuropsicológicos organizados em baterias fixas ou flexíveis. As baterias fixas são aplicáveis em pesquisas, em protocolos específicos para investigação de uma população particular. As baterias flexíveis são mais apropriadas para a investigação clínica, pois estão mais voltadas para as dificuldades específicas do paciente. Considerando a variação dos testes neuropsicológicos, tempo de aplicação e indicação, recomendamos organizar um protocolo básico com a possibilidade de complementar a avaliação com outros testes sobre as funções mais comprometidas, a fim de realizar um exame mais detalhado. A sensibilidade e a especificidade dos testes para as funções a serem examinadas deve ser considerada nesta escolha.

Portanto, de acordo com Maria Joana (2010), no livro avaliação neuropsicológica ${ }^{1}$, a avaliação neuropsicológica consiste no método de investigar as

\footnotetext{
${ }^{1}$ Malloy-Diniz, Leandro F. 2010.
} 
funções cognitivas e o comportamento. Trata-se de aplicação de técnicas de entrevistas, exames quantitativos e qualitativos das funções que compõem a cognição abrangendo processos de atenção, percepção, memória, linguagem e raciocínio. Há métodos considerados clássicos e outros ainda em construção. (JOAQUIM, 2010).

Dessa maneira é imprescindível destacar que a neuropsicologia utiliza-se dessa avaliação para diagnosticar as alterações comportamentais para fazer uma correlação com as lesões cerebrais que se apresentarem em seus pacientes.

E para isso é apontado um caminho que precisamos trilhar e assim se fazer inúmeros questionamentos, porque no final estaremos lidando com uma investigação sobre a memória de uma pessoa, onde ela é afetada por uma lesão cerebral e onde foram alteradas. Esse campo da neuropsicologia, essa ciência vai estudar essas relações entre o cérebro e o comportamento e especialmente as disfunções cognitivas decorrentes dessas lesões cerebrais.

Esse objetivo só se tornará possível mediante a aplicação de uma bateria de testes psicométricos que permitirão observar uma determinada função cognitiva e sua manifestação comportamental.

Ao ler o livro Avaliação Neuropsicológica publicado no ano de 2010, esse caminho é discutido criteriosamente por 104 autores de diferentes especialidades (psicólogos, psiquiatras, neurologistas, fonoaudiólogos, terapeuta ocupacional e pedagogos) e revela o caráter interdisciplinar da avaliação neuropsicológica. Cinco grandes temas são abordados em 49 capítulos, contribuindo de maneira significativa para uma melhor compreensão sobre a avaliação neuropsicológica no Brasil.

Assim sendo quando adentramos nesse caminho, descobre-se que o primeiro tema tratado no livro, psicometria, é uma introdução ao conceito de neuropsicometria que analisa conceitos como: modelo clássico e análise de Rasch (capítulo 01); os modelos monotético e idiográfico (capítulo 02) e as ferramentas para a elaboração de instrumentos de medida (capítulo 03).

O segundo tema, princípios gerais e domínios especificos, aborda as questões gerais e os princípios da avaliação neuropsicológica (capítulo 04) e análise de áreas específicas de investigação, tais como: o neuropsicólogo e seu paciente, inteligência 
geral, linguagem, memória, atenção, exame das funções executivas, praxia e visuoconstrução, matemática, dificuldade específica de aprendizagem da leitura e escrita, comportamento motor, cognição social, reconhecimento de emoções, teoria da mente, habilidades sociais, e a avaliação da personalidade e sua contribuição à avaliação neuropsicológica (capítulos 5 a 18).

O terceiro tema, ciclo vital, investiga a aplicação da avaliação neuropsicológica em diferentes períodos do desenvolvimento humano: idade pré-escolar, infância, adultez e no idoso (capítulos 19 a 23). O quarto tema, contribuições da avaliação para contextos específicos, analisa questões relacionadas à aplicação e a novas abordagens da avaliação neuropsicológica, tais como: psiquiatria e neurologia infantil, clínica neurológica de adultos e idosos, neurocirurgia, pesquisas em biologia molecular e em neurobiologia das funções cognitivas, práticas forenses, práticas esportivas e sua utilização na educação (capítulos 24 a 32).

O último tema, estudos no contexto brasileiro, é uma exposição sobre a utilização de diferentes testes neuropsicológicos no Brasil, destacando-se os seguintes testes: Teste de Atenção visual (TAVIS-3); Teste de Aprendizagem Auditivo Verbal de Rey (RAVLT); Teste da Torre de Londres (TOL); Teste da Torre de Hanói; Teste da Figura Complexa de Rey; Teste Bender (versão B-SPG); Nepsy II; Iowa Gambling Task e Children Gambling Task; Token Test; Bateria Memo, Questionário de Memória Retrospectiva e Prospectiva (PRMQ); Avaliação da Habilidade de Codificação Fonológica e Ortográfica; Avaliação Neuropsicológica de Processamento Lexical para Crianças; Escala de Avaliação de Demência (DRS); Matching Familiar Figures (MFFT-20); Teste de Organização Visual Hooper e Teste de Avaliação da Habilidade Visuoespacial (TAHLVES).

Só nessa leitura já podemos perceber a importância da avaliação neuropsicológica e todo o caminho que faremos ao entender dos temas tratados que são variados e abrangentes, descrevendo desde questões básicas até questões avançadas.

Entre outros temas, os estudos sobre as epilepsias e as demências têm muito contribuído para a investigação da sensibilidade e especificidade dos testes neuropsicológicos. 
A avaliação neuropsicológica nas demências tem por objetivos colaborar no diagnóstico diferencial entre demências, depressão e deficiência de memória associada à idade. Através da avaliação é possível determinar o nível atual de funcionamento, as características predominantes dos quadros demenciais. Demências consistem em distúrbios cognitivos, sensório-motores, das funções motoras e personalidade, suficientemente importantes para comprometer o funcionamento social e laboral do paciente. O diagnóstico é principalmente sintomatológico e a neuropsicologia tem uma participação importante neste. Dentre os testes mais sensíveis para detectar alterações precoces estão: Aprendizagem de Listas de Palavras, Figura Complexa de Rey (cópia e memória), Trail Making Test, Weigl Color Form Sorting Test, Fluência Verbal (Semântica e Categorias), e Semelhanças do WAIS R.

Atualmente temos poucos testes neuropsicológicos traduzidos e publicados no Brasil. A elaboração de um material próprio para nossa realidade pode ser bastante útil para a compreensão dos problemas específicos da população brasileira, mas dificultaria a análise destes resultados com base na literatura internacional. Quando o objetivo é o diagnóstico na clínica, é mais adequado utilizar técnicas bem respaldadas na literatura, mesmo que estrangeira, até que estejam disponíveis padronizações mais apropriadas.

Propomos como Protocolo Básico para Avaliação Neuropsicológica Clínica uma composição de testes de orientação, atenção, percepção, inteligência geral, raciocínio, memória verbal e visual, de curto e longo prazo, testes de flexibilidade mental, linguagem e organização visuo-espacial. O protocolo básico deve permitir ao examinador um panorama geral do funcionamento cognitivo do paciente, para posteriormente aprofundar sua avaliação com testes complementares. O resultado final deve fornecer um perfil neuropsicológico do paciente que, combinado a avaliação dos aspectos neurológicos, psicológicos e sociais, permitirá a orientação do paciente ou da família sobre o melhor aproveitamento de suas potencialidades. Conforme observamos o decorrer da história da neuropsicologia, os métodos utilizados variam de acordo com a formação de base do profissional e os locais de treinamento. 
Este deve também observar as particularidades de cada teste quanto a sua sensibilidade e especificidade, considerar os aspectos culturais e limitações do método utilizado.

Citando Kevin Walsh (1982) "na realidade, praticamente não existem testes neuropsicológicos, apenas o método de elaborar inferências sobre os testes é neuropsicológico". Sob este prisma, o mais importante o que avaliar e como avaliar. A avaliação neuropsicológica não é apenas a aplicação de testes e sim a interpretação cuidadosa dos resultados somada a análise da situação atual do sujeito e contexto em que vive. Somente com base nesta compreensão global é possível sugerir um diagnóstico.

O processo de avaliação inicia com uma entrevista clínica onde o histórico do paciente é investigado (escolaridade, ocupação, antecedentes familiares e história da doença atual) e esses parâmetros são utilizados na análise de resultados e na interpretação do impacto cognitivo das doenças neurológicas.

Por exemplo, um paciente que sofreu um trauma cranioencefálico pode apresentar disfunções, mas é necessário saber qual seu nível de funcionamento anterior para avaliar suas perdas. $^{2}$

Assim, podemos entender que enquanto técnica, a entrevista tem seus próprios procedimentos empíricos através dos quais não somente se amplia e se verifica, mas, também, simultaneamente, absorve os conhecimentos científicos disponíveis. Nesse sentido, Bleger (1980) define a entrevista psicológica como sendo "um campo de trabalho no qual se investiga a conduta e a personalidade de seres humanos". (BLEGER, 1980)

A avaliação neuropsicológica é capaz de revelar as alterações sutis, o nível de qualidade do funcionamento cognitivo. (GOTMAN, 1991). ${ }^{3}$ Para isso a avaliação neuropsicológica utiliza testes psicométricos e neuropsicológicos organizados em baterias fixas ou flexíveis.

\footnotetext{
${ }^{2}$ Malloy-Diniz, Leandro F. 2010.

${ }^{3}$ Malloy-Diniz, Leandro F. 2010.
} 
$\mathrm{Na}$ avaliação estruturada por meio de baterias fixas, ocorrerá geralmente dentro de uma demanda especifica. São muito úteis dentro do contexto de pesquisas ou serviços especializados em determinadas doenças neurológicas onde é necessária uma avaliação o mais formal possível.

A avaliação neuropsicológica nas demências tem por objetivos colaborar no diagnóstico diferencial entre demências, depressão e deficiência de memória associada à idade. Através da avaliação é possível determinar o nível atual de funcionamento, as características predominantes dos quadros demenciais. Demências consistem em distúrbios cognitivos, sensório-motores, das funções motoras e personalidade, suficientemente importantes para comprometer o funcionamento social e laboral do paciente.

A avaliação neuropsicológica desempenha um importante papel no diagnóstico diferencial entre o processo de envelhecimento cognitivo normal e patológico. A maior parte dos pacientes que progredirão para demência apresentarão sintomas compatíveis com Comprometimento Cognitivo Leve Amnéstico Único Domínio (CCLa UD) nos estágios iniciais da doença, que se diferencia do envelhecimento normal devido a uma evolução instável e declínios cognitivos severos (Charchat-Fichman, 2003), com comprometimento cognitivo progressivo e declínio funcional generalizado. Porém, nem todos aqueles que se encaixam no perfil de Comprometimento Cognitivo Leve (CCL) chegarão a desenvolver a demência, podendo haver ainda um diagnóstico. Dessa maneira, deve-se conhecer o perfil neuropsicológico de cada um dos subtipos para permitir identificá-los de maneira mais prática, o que só é possível através da avaliação neuropsicológica.

O diagnóstico é principalmente sintomatológico e a neuropsicologia tem uma participação importante neste. Dentre os testes mais sensíveis para detectar alterações precoces estão: Aprendizagem de Listas de Palavras, Figura Complexa de Rey (cópia e memória), Trail Making Test, Weigl Color Form Sorting Test, Fluência Verbal (Semântica e Categorias), e Semelhanças do WAIS R.

Dessa maneira a neuropsicologia vem contribuir com o seu papel de suma importância para o estudo da compreensão de como a mente funciona. E por outro lado 
a avalição psicológica baseada nessa abordagem explana os processos correlacionados com o funcionamento neurológico. Esses testes foram desenvolvidos para abordar algumas das áreas avaliadas pela neuropsicologia, especialmente dentro delas a memória e a atenção.

Alguns instrumentos têm também a sua contribuição para expandir a avaliação neuropsicológica, auxiliando na compreensão dos processos cognitivos e seus correlatos neurológicos, no aprimoramento de procedimentos de avaliação, e na promoção da reabilitação de indivíduos com disfunções ou lesões neurológicas, de forma responsável e eficiente.

Segundo Lezak et al. (2004), a avaliação neuropsicológica pode ser relevante para seis propósitos principais: a) diagnóstico; b) cuidados com o indivíduo; c) identificação de tratamentos necessários; d) avaliação dos efeitos de tratamentos; e) pesquisa e f) questões forenses.

Em relação ao diagnóstico, o avanço das técnicas de neuroimagem e dos exames laboratoriais diminuiu significativamente a necessidade da avaliação neuropsicológica para o diagnóstico da maior parte das lesões e disfunções neurológicas. Porém, esta avaliação ainda é crucial em determinados quadros, tais como as demências, traumatismos cranioencefálicos menores ou certas encefalopatias, visto que estes não são facilmente detectados nas técnicas usuais. Além disso, mesmo quando exames de neuroimagem detectam a presença de lesões, a avaliação neuropsicológica é fundamental para esclarecer os seus correlatos comportamentais, sendo ainda importante para o estabelecimento do prognóstico dos pacientes em determinados quadros e para a identificação precoce de certos distúrbios que, em seu estágio inicial, não apresentam alterações neurológicas óbvias.

Em relação aos cuidados com o indivíduo, a avaliação neuropsicológica pode fornecer, aos membros de seu convívio familiar e social, informações importantes relativas às suas capacidades e limitações. Estas informações incluem capacidade de autocuidado, capacidade de seguir o tratamento proposto, reações às suas próprias limitações, adequação de sua avaliação de bens e dinheiro, dentre outras. Conhecer estes aspectos do paciente é fundamental para estruturar o seu ambiente, promovendo 
alterações se necessário, de forma que ele tenha condições ótimas de reabilitar-se e evitando possíveis problemas secundários, como atribuição exagerada de responsabilidade ou de atividades que não estejam ao seu alcance.

Além de informações aos cuidadores, a avaliação neuropsicológica pode auxiliar o direcionamento da reabilitação, ao fornecer tantos dados sobre as áreas deficitárias do indivíduo, quanto sobre as habilidades preservadas e o potencial para a reabilitação. A avaliação serve, ainda, para verificar as mudanças do indivíduo ao longo das intervenções realizadas, sejam elas cirúrgicas, farmacológicas, psicológicas ou de outra natureza. Identificar tais mudanças, que podem ser positivas ou negativas, ajuda a rever as intervenções, redirecionando-as quando possível. 


\section{Capítulo II - O Comprometimento Cognitivo Leve}

Neste capítulo iremos entender a especificidade do que vem a ser o Comprometimento Cognitivo Leve (CCL), os critérios importantes para se fazer o diagnóstico e por último a compreensão do diagnóstico do Comprometimento Cognitivo Leve.

Algumas lacunas foram de tamanha importância para entendermos o que vem a ser o envelhecimento, etapa da vida do ser humano, acompanhada por altos níveis de doenças crônicas, quando não há uma qualidade de vida com condições saudáveis para o idoso. Outro ponto que destacamos é que a Organização das Nações Unidas (ONU) estabeleceu um marco de idade para a velhice que é a partir de sessenta anos.

Outro conceito de idoso é apontado como indivíduos denominados em um dado contexto sociocultural, em virtude das diferenças que exibem em aparência, força, funcionalidade, produtividade e desempenho de papéis sociais primários em comparação com adultos não idosos (NERI, 2009).

O Comprometimento Cognitivo Leve (CCL) é considerado por muitos autores como um quadro intermediário entre a cognição normal e a demência (PETERSEN, 2004; PETERSEN e cols, 1999). O diagnóstico precoce da doença de Alzheimer é fundamental, pois os pacientes com esta enfermidade serão os mais favorecidos por futuros tratamentos modificadores de doença, podendo protelar ou evitar o desenvolvimento de demência de Alzheimer nos pacientes com CCL (GAUTHIER; SCHELTENS; 2009).

Entretanto, o diagnóstico precoce ainda é um desafio. Muitos dos métodos atualmente em estudo utilizam tecnologias novas baseadas em biomarcadores, que atualmente apresentam elevado custo e baixa disponibilidade. Existe carência de métodos complementares de baixo custo e pouco invasivos (ALCOLEA e cols, 2014).

No Brasil, há carência de estudos que demonstrem a incidência, a prevalência, a evolução da cognição e a taxa de conversão dos pacientes com CCL para demência. Também há poucos estudos em nosso meio sobre os fatores de risco de progressão de 
CCL para demência. Além disso, a população brasileira possui a especificidade da baixa escolaridade entre os idosos, tornando-a distinta da maioria das populações atualmente estudadas e constituindo uma lacuna no conhecimento científico e elevando o interesse do estudo em nossa população (GUIMARÃES e cols, 2014; CÉSAR e cols 2016; GODINHO e cols, 2012; CHAVES e cols, 2009).

Portanto, o conceito de Comprometimento Cognitivo Leve (CCL) em idosos tem sido muito importante, porque esta condição se refere aos idosos que tem algum grau de perda cognitiva quando comparados a pessoas normais da mesma idade, mas não preenchem critérios de demência, pois alguns estudos comprovam que no processo de envelhecimento saudável observam-se alterações cognitivas leves envolvendo diminuição na velocidade do processamento de informação (MATTOS \& PAIXÃO JUNIOR, 2010).

É importante lembrar que este conceito se refere à fase de possível transição entre as modificações cognitivas esperadas para o processo de envelhecimento e um processo demencial, que compreenderia queixa subjetiva e prejuízo objetivo de funções cognitivas, especialmente memória episódica anterógrada. Nesta entidade diagnóstica, os idosos devem ter preservadas as atividades da vida diária e o funcionamento cognitivo global (PETERSEN, et al., 1999).

Entretanto, alguns estudos têm sugerido que o CCL pode representar um fator de risco para a Doença de Alzheimer (DA), pois a taxa de conversão para esta patologia de $10 \%$ a $15 \%$ ao ano, diferente com a de indivíduos normais em quem ela varia de $1 \%$ a $2 \%$ ao ano.

Entendemos que o envelhecimento normal pode ser acompanhado de um grau de declínio cognitivo, mas que não chega a interferir substancialmente com as atividades cotidianas. E esta alteração decorre de uma redução da velocidade de processamento de informações e de mudanças em certas habilidades cognitivas específicas, em especial a memória, a atenção, e as funções executivas.

Quando falamos sobre memória, na tabela abaixo, fica bem exemplificado para termos uma noção do que vem a ser memória para uma melhor compreensão. 
TABela 1

\begin{tabular}{|c|l|}
\hline Sistemas de Memória & \multicolumn{1}{|c|}{ Exemplos } \\
\hline Memória episódica & $\begin{array}{l}\text { L.embrar-se de uma pequena história, ou o que comeu no jantar na noite anterior, ou } \\
\text { o que fez no ültimo aniversảio. }\end{array}$ \\
\hline Memória semântica & Saber a cor de um leão. Saber a diferença entre um garfo e uma colher. \\
\hline Memória de procedimentos & Mudança de marchas ao dirigir um carro. Andar de bicicleta. t.er. \\
\hline Memória de trabalho & Realização de callculos matemáticos. \\
\hline Adaptada de Budson et al, 20054.
\end{tabular}

Assim quando se estuda o CCL entendemos que quando se observa a sua identificação, alguns autores seguiram os critérios de Petersen (2001), sendo estes, o autorretrato dos pacientes com relação ao prejuízo da sua memória.

As queixas subjetivas do idoso quanto à memória como critério diagnóstico não é confiável, pois é o que eles dizem sem qualquer critério, favorece assim o número de falsos estudos. Entretanto, outros estudos apontam que a percepção subjetiva não corresponde ao comprometimento objetivo da função e, isoladamente, não prediz o desenvolvimento de demência. Entretanto, a investigação da queixa subjetiva é fundamental em estudos em que o uso de testes psicométricos são limitados.

Elas acontecem em atividades cotidianas, relacionada a preocupações com a cognição, que pode estar associada ou não a déficits em testes objetivos. Estima-se que 17\% dos idosos apresentam queixas subjetivas da memória sem alteração em testes objetivos. Em metanálise publicada recentemente envolvendo pacientes com cognição normal sem queixa (controles) e com queixa subjetiva de memória (declínio cognitivo subjetivo) observou-se taxa anual de conversão para CCL de 6,6\% e para demência de $2,3 \%$ nos pacientes com queixa subjetiva, enquanto nos controles a taxa anual de conversão para demência foi de $1 \%$. Portanto, o declínio cognitivo subjetivo dobra o risco de desenvolvimento de demência entre idosos com cognição normal (MITCHELL e cols, 2014).

No entanto, não se conhece a taxa de conversão de Comprometimento Cognitivo Leve para demência de Alzheimer no Brasil, nem a influência de fatores descritos como 
de risco para conversão, assim como o diagnóstico de depressão e apatia. Conhecer este perfil de paciente em nosso meio, com nossas diferenças culturais e sociais em relação aos países mais desenvolvidos, é de extrema importância. Estas deficiências tornam o diagnóstico de CCL desafiador e limita a capacidade de no futuro ofertarmos tratamentos modificadores de doença aos pacientes do nosso meio.

Estima-se que o número de pessoas acometidas pela síndrome demencial no mundo era de 35,6 milhões em 2010 e que este número deve quase duplicar a cada 20 anos, elevando-se para 65,7 milhões em 2030 e 115,4 milhões em 2050 (PRINCE et al., 2013).

Estes autores apontaram que, em 2010, 58\% de todos os pacientes com demência viviam em países com rendimentos baixos ou médios (contexto no qual se insere o Brasil) e que essa proporção deve chegar a 63\% em 2030 e 71\% em 2050.

O crescimento dos casos de demência é tido como de caráter epidêmico e especialistas defendem que, se não controlado, representará enorme desestrutura para o sistema de saúde (WIMO \& PRINCE, 2010).

Só que em relação ao desempenho cognitivo como um todo, assim como o livro Neuropsicologia do envelhecimento, imagina-se que possa existir um continuum de perdas, que vai desde o conjunto de deficiências brandas descritas acima, até chegar aos quadros mais graves que caracterizam as síndromes demenciais. $\mathrm{O}$ principal divisor de águas, no caso da demência, é que essas perdas são suficientemente grandes para interferir na capacidade de executar as atividades de vida diária, levando, por último, à perda da autonomia e da independência. ${ }^{4}$

O Comprometimento Cognitivo Leve é classificado em dois subtipos principais: amnéstico e não amnéstico.

O subtipo amnéstico é o mais conhecido e faz referência a indivíduos com comprometimento da memória, especialmente a episódica, abaixo do esperado para a idade, podendo existir de forma isolada, neste caso sendo denominado CCL amnéstico único domínio, ou ser acompanhado por menores déficits cognitivos em outros

\footnotetext{
${ }^{4}$ Adaptado de Petersen, et al.
} 
domínios como linguagem, funções executivas, ou habilidades visuo-espaciais, neste caso chamado CCL amnéstico múltiplos domínios.

Já no CCL não amnéstico a memória está preservada, e como no anterior pode haver apenas um domínio comprometido ou múltiplos, então denominados CCL não amnéstico único domínio e CCL não amnéstico múltiplos domínios.

O CCL pode estar relacionado a uma heterogeneidade de condições clínicas, como o mal de Alzheimer, doença cérebro vascular, atrofia fronto-temporal, doenças cardiovasculares, trauma craniano, distúrbios metabólicos, e depressão entre outros. Alguns estudos mostram certa correlação entre o subtipo de CCL, suas possíveis etiologias e o tipo de evolução. Por exemplo, o CCL amnéstico, que é o mais citado na literatura sobre o CCL, tem uma presumível etiologia degenerativa, e os pacientes tenderiam a progredir para a doença de Alzheimer. ${ }^{5}$

A avaliação de diferentes habilidades cognitivas contribui para a identificação dos perfis neuropsicológicos e funcionais da transição entre o envelhecimento saudável e as síndromes demenciais (PAULA et al., 2010). Entretanto, em muitos contextos uma avaliação longa não é possível de ser realizada, ou não é a mais indicada. Nesse caso, buscam-se avaliações breves, de rápida e fácil aplicação. Nitrini et al. (1994), sugere, por exemplo, que para avaliações epidemiológicas, testes de aplicação rápida são os mais indicados, assim como em estudos que exigem amostras extensas, como estudos multicêntricos (MICHIELETTO, BINKIN, SAUGO, BOORSON \& SCANLAN, 2006). Também se considera que baterias rápidas facilitariam o exame e a triagem de pacientes (TAKESHI \& DODGE, 2010).

\section{1 - Alguns critérios importantes para fazer um diagnóstico}

O construto do Comprometimento Cognitivo Leve foi delineado ao final dos anos 90, como um quadro intermediário entre a cognição normal e a demência, com imenso interesse científico desde então, devido à futura possibilidade de prevenção secundária de estágios pré-clínicos das síndromes demenciais. Todavia, a existência desde quadro

\footnotetext{
${ }^{5}$ Adaptado de Petersen, et al.
} 
já era investigada muito antes da elaboração deste construto (PETERSEN, 2004; PETERSEN e cols, 1999).

Assim, a literatura descreve a doença de Alzheimer (DA) em três fases, a saber: a fase pré sintomática, a prodrômica (sintomática inicial) que inclui sintomas leves (CCL) e o estágio sintomático demencial. Provavelmente, esta classificação também é válida para as demências não-Alzheimer (DUBOIS e cols, 2007).

O primeiro critério diagnóstico amplamente utilizado foi o aplicado à corte de pacientes seguidos pela Mayo Clinic, essencialmente baseados no declínio da memória. Este declínio da memória é caracterizado por declínio de 1,5 desvio-padrão abaixo do esperado para a idade e escolaridade do indivíduo. As classificações diagnósticas de CCL divergem entre si sobre o grau de declínio dos domínios da cognição, variando de 1,0 a -1,5 desvios padrão (PETERSEN, 2004).

Reconheceu-se, posteriormente, que a memória não era o único domínio cognitivo afetado pelo CCL. (PETERSEN e cols, 2001). Petersen e cols (2004) propuseram novos critérios para CCL, essencialmente preservadas sem demência.

Em 2011, o National Institute on Aging (NIA) estabeleceu novas recomendações para o diagnóstico de CCL do tipo Alzheimer (amnéstico), mantendo os critérios clínicos semelhantes aos descritos por Petersen e cols (2004).

Segundo o NIA, o CCL devido à doença de Alzheimer (correspondente ao amnéstico) pode ser classificado como baseado somente em critérios clínicos (quando os biomarcadores não são realizados ou os seus resultados são conflitantes), probabilidade intermediária (realizado somente um tipo de biomarcador, que apresenta resultado positivo), probabilidade alta (os dois tipos de biomarcadores são positivos) ou improvável de ser devido à DA (similar ao não amnéstico, quando os resultados dos biomarcadores são negativos para $\beta$-amiloide e injúria neuronal), (ALBERT e cols, 2011). Em 2013, a American Psychiatric Association publicou o DSM-5, no qual o CCL foi reconhecido e denominado Transtorno Neurocognitivo Menor, com definição clínica próxima a descrita por Petersen e cols (2004) e Albert e cols (2011), com maior destaque a avaliação neuropsicológica e espaço para futura inclusão de novos métodos diagnósticos para diagnóstico etiológico. 
No ano de 2014, foi publicada uma revisão dos critérios diagnósticos do International Working Group (IWG) de 2007, os critérios do International Working Group 2 (IWG-2), com a definição de demência de Alzheimer, DA prodrômica e estágio pré-clínico da DA, com destaque para o comprometimento da memória e para os resultados dos biomarcadores. (DUBOIS e cols, 2004).

Portanto, há atualmente cinco critérios diagnósticos de CCL ou fase prodrômica de DA descritos na literatura, sendo dois voltados exclusivamente para a avaliação da presença de déficit amnéstico (embora a classificação do IWG-2 inclua, porém sem destacar, os demais domínios da cognição) e os outros três agregando declínio de qualquer domínio da cognição. (DUBOIS e cols, 2014; PETERSEN e cols, 2014).

\section{2 - O Diagnóstico de Comprometimento Cognitivo Leve}

O diagnóstico de CCL frequentemente é realizado em pacientes com alterações subjetivas de memória ou declínio cognitivo reportado por familiares. O paciente é submetido a métodos de triagem cognitiva que detecta déficits significativos de memória e/ou outros domínios cognitivos abaixo de pontos de corte estabelecidos para idosos de mesma idade e escolaridade. Entretanto, esta alteração não é suficiente para causar declínio funcional ou apenas suficiente para determinar declínios discretos em atividades de vida diárias avançadas. Este paciente, posteriormente, é submetido a métodos de imagem e/ou a análise de biomarcadores e/ou a avaliação neuropsicológica para confirmação diagnóstica (PETERSEN, 2004; ALBERT e cols, 2011; DUBOIS e cols, 2014).

Especificamente para o diagnóstico do Comprometimento Cognitivo Leve, a recomendação de 2011 pela Academia Brasileira de Neurologia, dividiu a análise da doença de Alzheimer em três fases: demência, comprometimento cognitivo leve e préclínica, sendo esta última somente para pesquisa clínica (FROTA et al, 2011).

Assim, o Comprometimento Cognitivo Leve pode ser avaliado por dois conjuntos de critérios: critério clínico central, para uso na prática clínica, sem necessidade de testes ou procedimentos altamente especializados e os critérios de pesquisa clínica que incorporam informações obtidas no uso de biomarcadores e destinadas à pesquisa em 
centros especializados. No caso do objetivo desse artigo, concentrar-se-á nos critérios para uso na prática clínica, pois, segundo Frota et al. (2011) pode utilizar o diagnóstico de CCL "em situações de pesquisas sem serem utilizados marcadores biológicos, buscando uma maior probabilidade de evolução para a DA", tornando-se, portanto, com um importante caráter preventivo da doença pelos neuropsicólogos.

São critérios para diagnóstico de CCL (FROTA et al, 2011):

- Queixa de alteração cognitiva relatada pelo paciente, informante próximo ou profissional;

- Evidência de comprometimento cognitivo em um ou mais domínios cognitivos; tipicamente incluindo a memória, obtida através de avaliação que compreenda os seguintes domínios cognitivos: memória, função executiva, linguagem e habilidades visuais-espaciais; ou exame neuropsicológico;

- Preservação da independência nas atividades funcionais. Pode haver problemas leves para executar tarefas complexas anteriormente habituais, tais como pagar contas, preparar uma refeição ou fazer compras. O paciente pode demorar mais, ser menos eficiente e cometer mais erros ao executar essas atividades. No entanto, ainda é capaz de manter sua independência com mínima assistência. Assim, para avaliação do estado mental e rastreio cognitivo na detecção de DA a Academia Brasileira de Neurologia $(\mathrm{ABN})$ recomenda a utilização do Mini-Exame do Estado Mental (padrão). Podendo, ainda, serem utilizados outros instrumentos como o CASI-S, a Bateria Breve de Rastreio Cognitivo e o Exame Cognitivo de Addenbrooke-R podem ser utilizados ampliando o escopo da avaliação cognitiva.

Apesar da indicação pela Academia Brasileira de Neurologia, foi realizada uma pesquisa para verificação se tais instrumentos foram validados pelo Conselho Federal de Psicologia. A pesquisa foi realizada no site do conselho, utilizando-se do sistema Satepsi - Sistema de Avaliação de Testes e Instrumentos Psicológicos. Como resultado foi constatado que nenhum dos testes e instrumentos recomendados pela $A B N$ foi aprovado ou recomendado pelo Conselho Federal de Psicologia. É verdade que o Mini Exame do Estado Mental, recomendado pela $\mathrm{ABN}$, pode ser aplicado por outros profissionais, inclusive neuropsicólogos, mas não há indicação pelo CFP que os 
psicólogos podem utilizar tal instrumento na avaliação psicológica, trazendo uma questão a ser refletida: poderá o profissional de psicologia, mesmo os que atuam como neuropsicólogos, validarem tais testes e utilizarem o seu número de CRP para validar tais resultados?

A área de neuropsicologia vem, entretanto, utilizando alguns dos testes aprovados pelo CFP combinados ao Mini Exame do Estado Mental para realizar diagnósticos de comprometimento leve:

Segundo Handam (2008) os testes mais empregados no diagnóstico de DA são o Mini Exame do Estado Mental (MEEM), as escalas funcionais, como a de Pfeffer e o Teste do Desenho do Relógio (TDR).

O MEEM avalia as dimensões em relação à orientação no tempo, no espaço, de registro, de atenção de cálculo, de memória de evocação e de linguagem. A escala de Pfeffer é composta por dez itens que evidencia a funcionalidade através do grau de independência para realização das atividades instrumentais de vida diária.

O teste do desenho do relógio objetiva avaliar a percepção do paciente em relação aos horários, assim como o planejamento, atenção na execução motora, percepção visual, coordenação visomotora e capacidade visuo-constritiva. Albanos (2009) efetuou uma análise correlacionando o Mini-Exame do Estado Mental (MEEM) com outros instrumentos como: o Teste do Desenho do Relógio, a Bateria Rápida de Avaliação Frontal (BREF), o Escore Clínica de Demência (CDR), a Escala de Depressão Geriátrica - versão curta (EDG), o de Atividades da Vida Diária (AVKATZ), Inventário de Atividades da Vida Diária (AIVDLAWTON), o Queixas Mnésicas para Fatos Recentes (MacNair-Pc; MacNair- Ac) e o Teste de Semelhanças (TS).

Além dos testes, foram analisados também aspectos sociais, como: escolaridade, idade, tabagismo/etilismo, atividade física, etc. Os resultados demonstraram que o MEEM possui boa correlação com todos os testes, mas ainda não distinguia uma demência da outra e que, assim, seria necessário o auxílio de outros testes específicos.

Outra pesquisa, publicada por Schlindwein-Zanini (2010), realizou uma avaliação neuropsicológica abreviada constituída pelo Mini-exame do Estado Mental, o Teste do Desenho do Relógio, o Teste de Fluência Verbal, o Questionário de Atividades 
Funcionais (Pfeffer) e a Escala para Depressão Geriátrica (EDG), combinando instrumentos da Neurologia com os da Psicologia.

Segundo Montiel et al. (2014) o teste do desenho do relógio (TDR) e de fluência verbal (FV) são de simples aplicação e amplamente utilizados em avaliações neuropsicológicas que procuram investigar síndromes demenciais em idosos

Não existem ainda testes neuropsicométricos específicos para a avaliação cognitiva no CCL, devendo a mesma ser realizada pela investigação dos domínios cognitivos específicos envolvidos, tais como a atenção, a memória e as funções executivas. As informações obtidas são úteis tanto no diagnóstico do comprometimento, como no seu seguimento pelo controle da progressão dos déficits, com exames seriados.

É importante frisar que todos os testes têm alta taxa de resultados falso-negativos quando aplicados em uma fase muito inicial de alterações cognitivas, ou em indivíduos com inteligência prévia elevada, e também de falso-positivos em indivíduos normais com baixa escolaridade, condição comum em nosso meio. 


\section{Capítulo III - O Comprometimento Cognitivo Leve e a Avaliação Neuropsicológica}

Este capítulo é determinado como é feito uma avaliação neuropsicológica em um paciente com diagnóstico do Comprometimento Cognitivo Leve, não precisamos dos resultados desta pesquisa, mas sim do método e também é preciso esclarecer que há algumas diferenças dos subtipos, o desempenho objetivo de testes neuropsicológicos que tem sido a metodologia utilizada para validar o critério clínico de Comprometimento Cognitivo Leve, definido como comprometimento em uma ou mais funções cognitivas com a preservação das atividades funcionais (FROTA et al., 2011). Este critério é fundamental para a identificação da ausência ou presença de CCL, bem como estabelecer os subtipos específicos com risco diferencial para progressão em síndromes demenciais (PETERSEN et al., 2014).

Para isso, entendemos aqui que o Comprometimento Cognitivo Leve (CCL) corresponde a uma provável transição para demência e seu diagnóstico pode promover uma intervenção precoce tanto medicamentosa quanto comportamental (PETERSEN, et al., 2009). A avaliação neuropsicológica desempenha um importante papel no diagnóstico diferencial entre o processo de envelhecimento cognitivo normal e patológico (DE PAULA et al., 2013).

\section{1 - Os subtipos Amnéstico e não Amnéstico}

Os subtipos de Comprometimento Cognitivo Leve, dividem-se basicamente entre amnéstico e não amnéstico.

O CCL amnéstico (CCLa) caracteriza-se pelo declínio da memória isolada (de único domínio - UD) ou juntamente com outra função cognitiva (múltiplos domínios MD) (ZHENG et al., 2012). Além disso, observa-se uma ausência de demência e de dificuldades para a realização das atividades de vida diária (AVDs).

O CCL não amnéstico (CCLna) pode ser de UD quando se refere a pacientes com comprometimento em um único domínio cognitivo, com exceção da memória, ou de 
MD, quando há comprometimento em 2 ou mais domínios, também com exceção da memória (Charchat-Fichman et al., 2013).

Segundo Forlenza, Diniz, Stella, Teixeira e Gattaz (2013) a maior parte dos pacientes que progredirão para demência apresentarão sintomas compatíveis com CCLa UD nos estágios iniciais da doença, que se diferencia do envelhecimento normal devido a uma evolução instável e declínios cognitivos severos (Charchat-Fichman, 2003), com comprometimento cognitivo progressivo e declínio funcional generalizado. Porém, nem todos aqueles que se encaixam no perfil de CCL chegarão a desenvolver a demência, podendo haver ainda super diagnósticos.

Dessa maneira, deve-se conhecer o perfil neuropsicológico de cada um dos subtipos para permitir identificá-los de maneira mais prática, o que só é possível através da avaliação neuropsicológica.

Apesar da relevância da avaliação neuropsicológica, existem controvérsias na literatura quanto a alguns critérios metodológicos como: 1) escolha dos instrumentos mais adequados para o diagnóstico de CCL; 2) variação dos critérios utilizados para classificar o comprometimento: ponto de corte ou desvio-padrão; 3) estabelecimento de algoritmos diferentes; 4) diferenças etárias, sociais, educacionais e demográficas entre amostras que dificultam as comparações de estudos; 5) influência de variáveis sócio demográficas, como idade e escolaridade, no desempenho dos testes; e 6) número escasso de instrumentos validados, normatizados e padronizados para a população idosa brasileira (Clark et al., 2013; Damin, 2011; de Paula et al., 2013; Forlenza, Diniz, Stella, Teixeira, \& Gattaz, 2013; Ladeira, Diniz, Nunes, \& Forlenza, 2009; O'Caoimh et al., 2013; Petersen et al., 2009; Petersen et al., 2014; Yassuda et al., 2009; Winblad et al., 2004).

De acordo com a literatura brasileira e internacional, alguns testes como o Teste de Fluência Verbal (TFV) e o Teste do Desenho do Relógio (TDR), componentes da Bateria Breve de Rastreio Cognitivo (BBRC), teriam propriedades psicométricas apropriadas para a avaliação de idosos com baixa escolaridade (de Paula et al., 2013; Jacinto et al., 2014; Ladeira, Diniz, Nunes, \& Forlenza, 2009; Malloy-Diniz et al., 2000; Nitrini et al., 2005; Salmon \& Bondi, 2009). 
Porém, o ideal é que a avaliação neuropsicológica seja mais extensa e específica que as de rastreio. Nesse caso, foram escolhidos para o estudo, a Escala Mattis de Avaliação de Demência (EMAD) e o Teste de Aprendizagem Auditivo-Verbal de Rey (TAAVR).

A EMAD é muito utilizada para a detecção precoce de demência e diagnóstico diferencial entre DA e outros tipos de demência (Marson et al., 1997; Monsch, et al., 1995; Paolo et al., 1995; Porto et al., 2003), tendo apenas em 2013 expandidas suas normas para a população brasileira por idade e escolaridade (Foss et al., 2013). O TAAVR, elaborado por Rey em 1985 e, apenas em 2000, tendo sido desenvolvidas normas para a população brasileira: dos 16 aos 89 anos de idade (Malloy-Diniz, Lasmar, Gazinelli, Fuentes, \& Salgado, 2007), é mais específico para o diagnóstico diferencial (Cotta et al., 2012) e avalia os processos de aprendizagem, evocação e reconhecimento da memória episódica, além da memória de curto prazo e distração (Diniz, Cruz, Torres, \& Cozensa, 2000). Controvérsias metodológicas nos estudos com CCL dificultam sua definição clínica (Charchat-Fichman, 2003; Clark et al., 2013; de Paula et al., 2013; Foss, Vale, \& Speciali, 2005).

Um problema refere-se ao número reduzido de instrumentos validados, normatizados e padronizados para populações com heterogeneidade sócio demográfica a exemplo da brasileira e de outros países da América Latina. Ausência de definição de critérios de normalidade e comprometimento para idosos. Diante destas questões, o objetivo deste estudo foi investigar o papel da avaliação neuropsicológica na definição do conceito de Comprometimento Cognitivo Leve (CCL) e identificar seus perfis neuropsicológicos baseados no uso de um algoritmo cognitivo. Diante da dificuldade de definir critérios neuropsicológicos para o diagnóstico de CCL, especialmente para populações clínicas brasileiras, e devido à heterogeneidade do nível de escolaridade e do perfil socioeconômico, ausência de instrumentos validados, normatizados e padronizados, ausência de uma definição de normalidade e comprometimento para a população (CharchatFichman, 2003; Clark et al., 2013; de Paula et al., 2013; Foss, Vale \& Speciali, 2005), o objetivo deste estudo foi investigar o papel da avaliação neuropsicológica na definição do conceito de Comprometimento Cognitivo Leve (CCL) e identificar seus perfis neuropsicológicos baseados no uso de um algoritmo cognitivo. 
Para investigar o papel da avaliação neuropsicológica na definição do conceito de Comprometimento Cognitivo Leve (CCL) e identificar seus perfis neuropsicológicos, foram avaliados 88 idosos atendidos em um hospital do Rio de Janeiro.

Todos os pacientes foram classificados como CCL a partir da avaliação geriátrica e de seu desempenho na Bateria Breve de Rastreio Cognitivo (BBRC). Idosos diagnosticados com demência pela equipe médica foram excluídos do estudo.

A fim de discutir o papel da avaliação neuropsicológica no conceito de CCL, os pacientes foram avaliados pela Escala Mattis de Avaliação de Demência (EMAD) e pelo Teste de Aprendizagem Auditivo-Verbal de Rey (TAAVR). Um algoritmo cognitivo foi proposto e os idosos com CCL foram classificados nos seguintes perfis neuropsicológicos: 1) preservação das funções cognitivas (normal), 2) amnéstico único domínio, 3) amnéstico múltiplos domínios, 4) não-amnéstico e 5) amnéstico múltiplos domínios prejudicando o funcionamento cognitivo global (Doença de Alzheimer - DA). Resultados: O perfil neuropsicológico DA (33\%) e não-amnéstico (33\%) foram os mais frequentes seguidos dos amnésticos (20\%) e normal (14\%). O perfil DA apresentou comprometimento na EMAD Total, bem como em todas as outras sub escalas da EMAD e variáveis do TAAVR, o perfil não-amnéstico comprometimento predominante da EMAD Construção e o amnéstico comprometimento da EMAD Memória e Evocação Livre após intervalo TAAVR A7. As variáveis que melhor discriminam os perfis foram A7 e EMAD Construção, de acordo com a ANOVA seguida por post-hoc Bonferroni. 


\section{Considerações finais}

Por derradeiro, manifesto um profundo sentimento de realização ao concluir esse trabalho, tanto pela escolha desse tema que veio ao encontro da minha experiência vivida no Serviço de Psicologia Aplicada da Pós-graduação, quanto pelo robustecimento de meus conhecimentos obtidos no estágio supervisionado.

Terminado esse estudo, diante do que foi visto e revisto é necessário fazer as considerações acerca de todo o assunto e na tentativa de tecer nesse trabalho alguns apontamentos dos aspectos mais importantes para a avaliação neuropsicológica e o diagnóstico diferencial que aponta certas dificuldades metodológicas no diagnóstico de CCL.

Considerar apenas a dicotomia do perfil amnéstico e perfil não amnéstico pode negligenciar outros déficits que não envolvam a memória (linguagem, função executiva e habilidades visuoespaciais, atenção, entre outras) (CLARK et al., 2013).

Lembramos que a revisão bibliográfica permitiu clareza no desenrolar de várias questões, inclusive do objetivo a ser alcançado, o de compreender a avaliação neuropsicológica sob o olhar da neuropsicologia como uma ferramenta essencial para mensurar o declínio das funções cognitivas.

A partir da fundamentação teórica, pode-se pensar que cada autor tem sua visão sobre o CCL, com suas características e diferenças, mas, quando analisadas mais profundamente complementam-se.

Nas etapas dos testes que contribuem de forma diferenciada para explorar a heterogeneidade cognitiva dos idosos diagnosticados com CCL é bom pensar que os testes mais abrangentes minimizam o efeito dos falsos negativos esperados quando se utiliza testes de rastreio.

Dessa forma, podemos concluir que este trabalho possibilitou a compreensão do CCL não apenas como um estágio de transição para a demência, mas como uma síndrome clínica heterogênea e com perfis cognitivos diferenciados. Há diversas possibilidades e definições de comprometimento cognitivo. Dependendo de quais testes 
e como se delineia o algoritmo, determinadas especificidades são propostas. Conhecer as funções cognitivas mais comprometidas no processo de envelhecimento e no CCL permite que se defina um algoritmo mais próximo da realidade. A padronização de um algoritmo pode auxiliar as próximas pesquisas e a prática clínica, especialmente em países da América Latina como o Brasil com uma população idosa heterogênea em relação ao nível de escolaridade e reserva cognitiva.

Dito isso, a importância no desenvolvimento da CCL é um elo entre o envelhecimento e o seu mundo, diferente de ser somente uma forma subjetiva do idoso é também uma maneira de expressar o processo e de tudo que afeta essa etapa da vida do ser humano. 


\section{Referências Bibliográficas.}

AURÉLIO. Minidicionário da língua portuguesa. Rio de Janeiro: Nova Fronteira, 1993.

BLEGER, José. Temas de psicologia: entrevista e grupos. Trad. Rita M. de Moraes. São Paulo: Martins Fontes, 1980.

MATTOS, P., \& PAIXÃO J., C.M. (2010). Avaliação cognitiva de idosos: envelhecimento e comprometimento cognitivo. In L.F. Malloy-Diniz, D. Fuentes, P. Mattos, N. Abreu. (Orgs.), Avaliação neuropsicológica (pp. 247253). Porto Alegre: Artmed.

Zibetti, M. R., Gindri, G., Pawlowski, J., de Salles, J. M., Parente, M. A. M. P., Bandeira, D. R., Fachel, J. M. G., \& Fonseca, R. P. (2010). Estudo comparativo de funções neuropsicológicas entre grupos. Revista Neuropsicologia Latinoamericana,

MALLOY-DINIZ, C. H. P. Camargo, \& R. M. Cosenza (Orgs.), Neuropsicologia: Teoria e prática (2. ed.). Porto Alegre: Artmed.

FUENTES, D., MALLOY-DINIZ L. F., CAMARGO, C. H. P., COSENZA, R. M. et. al. (2008). Neuropsicologia - Teoria e Prática. Porto Alegre: Artmed.

TABAQUIM, M. L. M. (2003). Avaliação neuropsicológica nos distúrbios de aprendizagem. Em: Sylvia Maria Ciasca (org.). Distúrbios de aprendizagem: proposta de avaliação interdisciplinar. São Paulo: Casa do Psicólogo.

THIERS, V. O., ARGIMON, I. L. \& NASCIMENTO, R. F. L. (2005) Neuropsicologia: A expressão comportamental dos processos mentais. (WWW.psicologia.com.pt).

VENDRELL, J. M. (1998). A evolução da ciência neuropsicológica e sua importância no mundo atual. In F. C. Capovilla, M. J. Gonçalves \& E. C. Macedo (Eds.), Tecnologia em (re) habilitação cognitiva: uma perspectiva multidisciplinar (pp. 19-26). São Paulo: Edunisc \& SBNp

MALLOY-DINIZ, L. et al. . Avaliação Neuropsicológica. Porto Alegre: Artmed, 2015.

MALLOY-DINIZ, L. et al. Neuropsicologia das Funções Executivas. Em: MALLOYDINIZ et al., Neuropsicologia - Teoria e Prática (pp.187-206). Porto Alegre: Artmed, 2008.

MALLOY-DINIZ, L. F. I.; FUENTES, D.; COSENZA, R. M. (Org.) Neuropsicologia do Envelhecimento: Uma Abordagem Multidimensional. Porto Alegre: Artmed, 2013. 
NERI, A. L (ORG.) Desenvolvimento e Envelhecimento: Perspectivas Biológicas, Psicológicas e Sociológicas. 3ed. Campinas, SP: Papirus, 2007.

ORGANIZAÇÃO MUNDIAL DE SAÚDE (OMS). Demência: Um caso de saúde pública. Geneva: WHO, 2012.

ORGANIZAÇÃO MUNDIAL DE SAÚDE (OMS). OCID-10: Classificação internacional de doenças mentais e comportamentais. Critérios diagnósticos. Genebra: Organização Mundial de Saúde, 1993.

\section{ACESSO A LINKS:}

Revista Neuropsicologia Latinoamericana file://C:/Users/fer_c/Desktop/2571743-4-PB.pdf. Acesso em: 09/08/2018.

Artigo Perfis neuropsicológicos do Comprometimento Cognitivo Leve no envelhecimento (CCL) é de Helenice Charchat Fichman (2015). Acesso link: http://pepsic.bvsalud.org/scielo.php?script=sci_arttext\&pid=S207594792015000 200002 em 13/09/2018. 